\title{
Protocol for a rapid evidence review of traditional and complementary medicine for people with diabetes receiving palliative or end-of-life care
}

\begin{abstract}
Background: Rapid review methods are increasingly used as an alternative to systematic reviews when there is time, resource or other logistical constraints. The Cochrane Rapid Reviews Methods Group recommends further methodological development and publishing protocols to improve the transparency and quality of the review. The authors of this paper were invited to provide timely, expert input for the revision of the 2010 Guidelines for Providing Palliative and End of Life Care for People with Diabetes (Guidelines), Australia, regarding the evidence-based use of traditional and complementary medicine (T\&CM). The inclusion and consideration of T\&CM in guidelines is often ad-hoc and would benefit from more systematic methods. Evidence of efficacy on clinical outcomes is not the only reason to review an intervention. Other reasons relevant to the T\&CM context is: interventions that are commonly used by people with diabetes in the palliative care setting, have potentially high costs or risks, or present a conflict in choices between individual and societal perspectives.
\end{abstract}

Method: The aim this review is to rapidly identify and synthesise the highest quality evidence about the safety and efficacy of a selection of T\&CM interventions and provide timely information to update the Guidelines. The review has two stages 1) a rapid scoping review to inform the framing of the review questions and identify important modifying factors and 2) a rapid evidence review of up to $20 \mathrm{~T} \& \mathrm{CM}$ interventions to inform the Guidelines. Project constraints include limiting the number of interventions for appraisal and databases to be searched, and including only papers published in English. Searches and evidence appraisals will be conducted by single reviewers, with one tenth to be checked by another reviewer. No meta-analyses or modelling will be undertaken.

Discussion: The proposed rapid review protocol is designed to address the time and resource constraints of the Guidelines developers, and inform rapid review methodology. Notwithstanding the methodological constraints, the proposed protocol and its reporting will be transparent, systematic and reproducible.

Protocol Review: This protocol is endorsed by the Guidelines' Writing Group and the Health Professional Expert Advisory Group.

Keywords: diabetes, end of life, palliative care, complementary medicine, integrative medicine, clinical guidelines, rapid review, scoping review, literature review
Volume I Issue 7 - 2017

\author{
Jennifer Hunter, ${ }^{1,2}$ Susan Arentz,' Gary \\ Deed 1,3 \\ 'School of Science \& Health, Western Sydney University, \\ Australia \\ ${ }^{2}$ Menzies Centre for Health Policy, University of Sydney, \\ Australia \\ ${ }^{3}$ Mediwell Coorparoo, Australia
}

\begin{abstract}
Correspondence: Jennifer Hunter, MScPH, BMed, NICM, School of Science \& Health, Western Sydney University, Campbelltown Campus, Locked Bag 1797, Penrith NSW 275I, Australia, Email jennifer.hunter@westernsydney.edu.au
\end{abstract}

Received: November 07, 2017 | Published: December 27, 2017
Abbreviations: T\&CM, traditional and complementary medicine; PICO, patient/population/problem, intervention, comparison, outcome; UK, united kingdom; USA, united states of America; SR, systematic reviews; AMED, allied and complementary medicine database

\section{Introduction}

This paper presents a protocol using rapid review methods that will be used to select a limited number of traditional and complementary medicine (T\&CM) interventions and evaluate the available evidence. The results from this review will be used to inform an update of the Australian Guidelines for Managing Diabetes at the end of life (Guidelines). Rapid review methods were chosen to maximise the number of T\&CM interventions and different problems that could realistically be reviewed in a timely manner given the resources available to the research team.

Rapid review methods are increasingly used as an alternative to systematic reviews (SR) when there are time, resource or other logistical constraints. ${ }^{1-5}$ Estimated costs of a comprehensive SR exceed USD $\$ 100,000$ with an average of 1,139 hours to complete. ${ }^{6}$ For many research teams, pragmatic decisions must be made that may compromise the accepted 'gold standard' systematic review methods used to minimise bias, which in turn impacts the validity and generalisability of the results. As such, a rapid review is not a replacement for a full systematic review and meta-analysis. Notwithstanding methodological constraints, rapid reviews should endeavour to be transparent, systematic and reproducible. ${ }^{4}$ Most Rapid Reviews do not publish a protocol. ${ }^{3}$ However, the recent establishment of a Cochrane Rapid Reviews Methods Group highlights the need to develop methods that minimise bias despite project constraints. Outlining the objectives and methodology a priori will further improve the transparency and quality of the methods used to frame the review questions, help reduce bias arising from the rapid review study design, and enhance the reproducibility of the results. 
Diabetes mellitus is a common end-of-life condition due to its high prevalence and associated complications and co-morbidities. In 2012, $10 \%$ of Australian death certificates recorded diabetes as an underlying or associated cause of death. Mortality rates were 1.6 times higher for males than females, and exponentially increased with age. ${ }^{8}$ Palliative and end of life care are important aspects of the diabetes care. The trajectory to death for people with diabetes is usually long with many periods of instability before death occurs; but death can be sudden. For others, diabetes only becomes an issue at the end of life when glycaemic control is exacerbated by medications being used to manage another condition. Palliative and end of life care for people with diabetes involves management decisions that emphasise enhanced person-centred quality-of-life over curative treatments. ${ }^{9}$ The importance of early end-of-life planning is increasingly being recognised, including the potential for cost savings with Advance Care Planning programs. ${ }^{10}$

T\&CM covers a diverse range of health-related interventions and therapies that are typically not considered to be part of conventional Western medical care (Table 1). ${ }^{11-13}$ The decision to include guidance about the use of T\&CM in the Guidelines reflects their high use by people with diabetes, ${ }^{14-16}$ the provision of selected T\&CM interventions by hospices and palliative care services, ${ }^{17-19}$ the potential for both negative and positive interactions with conventional treatments, ${ }^{20}$ and the alignment of many of the philosophies and aims of T\&CM with palliative care, such as relieving suffering and a focus on holistic, person-centred care..$^{21,22}$ Inclusion of T\&CM in the Guidelines aims to better support the need for informed health care communication and decision-making, ${ }^{23}$ and contribute to coordinated continuity of care across multiple healthcare settings - hospital, home, long-term care and hospice.

Table I Traditional \& Complementary Medicine (T\&CM) therapies and practices

\section{Mind-body medicine}

\section{Natural product based} therapies and diets

\section{Manipulative and body-} based practices

\section{Energy medicine}

\section{Whole medical} systems
Techniques to enhance the mind's capacity to affect bodily function and symptoms and vice versa e.g. play, art or music therapy, aromatherapy, hypnosis, imagery, tai chi, yoga, meditation.

Substances found in nature to promote health (e.g. nutritional supplements, plant based medicines, chelation therapy) and diet therapy (e.g. low glycaemic-index diet, vegetarian or vegan diet).

Hands-on manipulation and/or movement of parts of the body such as chiropractic, osteopathy, massage, reflexology.

The use of energy fields, including the unconventional use of electromagnetic fields, that purportedly surround and penetrate the human body e.g. acupuncture, laser acupuncture, magnetic therapy, therapeutic touch, Reiki.

Which are complete systems of theory and practice outside the conventional, allopathic model e.g. traditional Chinese medicine, Ayurvedic medicine, Naturopathy, Homeopathy and Indigenous healing practices.
There is a paucity of evidence to guide clinical decision-making for diabetes palliative and end of life care in this clinical context that in part reflects the ethical challenges with recruiting vulnerable people. ${ }^{24}$ Notwithstanding, evidence-based guidelines have been produced by various bodies in the UK, USA and Australia. ${ }^{25,26}$ However, the guidelines mostly focus on the management of diabetes and glycaemic control with limited discussion about the management during the palliative care stages of a person who already has, or develops diabetes. ${ }^{27}$ Furthermore, T\&CM use is rarely considered in guidelines for diabetes or end of life care, and a common complaint of guidelines in general, is the ad-hoc inclusion and consideration of $\mathrm{T} \& \mathrm{CM} .^{28,29}$

In response to the need for clinical guidance leading Australian experts published the Guidelines for Managing Diabetes at the end of life (Guidelines) in 2010. ${ }^{30}$ The Guidelines are now due for revision. Along with revising the existing recommendations, the proposed revision will expand the scope to include palliative care, include more information about common disease trajectories and communication processes to promote earlier recognition and discussion about end-oflife planning, and provide more specific recommendations about the appropriate use of T\&CM.

Regarding the decision about which of the many different T\&CM interventions to appraise, GRADE and other directives for guideline developers recommend formulating the PICO questions - Patient/problem/population, Intervention, Comparison interventions and Outcomes - through consensus development by the guideline committee. ${ }^{31,32}$ However, non-systematic methods such as brainstorming are often recommended. ${ }^{33}$ The concern is that brainstorming will reflect the expert knowledge and bias of the committee members, and may in part explain the ad-hoc inclusion of $\mathrm{T} \& \mathrm{CM}$ in guidelines. ${ }^{34} \mathrm{~A}$ systematic method is therefore needed when framing the PICO questions for T\&CM. ${ }^{34}$ Evidence about safety, efficacy and cost-effectiveness, and the potential for at least a modest clinical benefit are important, but not the only reasons, to appraise an intervention. Other reasons relevant to the T\&CM context includes interventions that are commonly used by people with diabetes, are commonly provided in the healthcare setting, have potentially high costs or risks, or present a conflict in choices between individual and societal perspectives. ${ }^{35,36}$

Another common criticism of guidelines, is that due to the emerging scientific evidence-base for many T\&CM interventions, recommendations regarding their use are often non-specific such as "discuss T\&CM use with your patients", or "more research is needed". ${ }^{28,29,37}$ Neither is particularly informative to patients and practitioners who must make informed decisions despite insufficient high-quality evidence. The importance of providing informative recommendations is emphasised in many guidelines directives. ${ }^{38-41}$ Evidence about the benefits and risks of an intervention is only part of the information required to make informed clinical decisions. Modifying factors such as patient preferences and values; affordability, accessibility, quality and feasibility of the intervention; the health care setting; and other social and economic circumstances must also be considered. ${ }^{36,42,43}$ Consequently, the use of an evidence hierarchy as a direct constrainer when grading the strength of a recommendations is now discouraged ${ }^{44}$ as there is a tendency for guideline developers to then ignore lower levels of evidence and important modifying factors that in some instances, may justify upgrading a recommendation. ${ }^{38}$ 
In summary, the challenges with including T\&CM interventions in palliative and end of life clinical guidelines are:

i. Selecting which of the many T\&CM options to include in an in-depth evidence review;

ii. Finding and appraising the evidence of safety, efficacy and costeffectiveness, along with information about relevant modifying factors such as the magnitude of effect, current use, patient preferences, demand, equity and logistical considerations (e.g. quality of products, credentialing and availability of practitioners, implementing the service);

iii. Providing useful, specific recommendations despite the likelihood that for many interventions there will be uncertainty about safety or efficacy; and

iv. Obtaining the information in a timely manner despite resource constraints.

Despite these challenges, the authors of this paper were invited to provide expert input about the evidence-based use of T\&CM in diabetes end-of-life care for the revised Guidelines. Following a series of meetings with the Guidelines' Writing Group and Health Professional Expert Advisory Group that included discussions about time and resource constraints, the decision was made to conduct a rapid literature review to frame the questions, and appraise and summarise the evidence for up to $20 \mathrm{~T} \& \mathrm{CM}$ interventions. The results will be used to inform the Guidelines and provide relevant information to clinicians and people with diabetes.

\section{Materials and methods}

It is recommended that the use of rapid reviews are restricted to answering narrow, focused questions in a timely manner, or used as a scoping mechanism for emerging technologies such as T\&CM. ${ }^{5}$ In this instance, both reasons apply. The proposed rapid review has two parts:

i. A scoping review to frame the review questions and identify important modifying factors and

ii. An evidence review of selected T\&CM interventions.

\section{Project constraints}

This review is constrained by both time and resources. The review must be accelerated and completed within 6 months. Although there are three reviewers, their availability is limited to a total of 0.2 FTE (full time equivalent) for the duration of the project. As such, the following constraints are proposed. The literature searches will be restricted to the smallest number of English language databases possible. The assumption is that if the majority of relevant publications are identified, inclusion of the remaining studies is unlikely to substantially change the estimates of effect. ${ }^{45}$

One reviewer will conduct each search and select eligible publications. Similarly, the methodological quality of the included studies will be formally appraised by one reviewer only, who will refer to the other reviewers for a second opinion if there is doubt, including equivocal evidence. As a means of monitoring quality and consistency between the reviewers, one tenth of the publications will be randomly selected throughout the process for appraisal by another reviewer in the research team. Significant discrepancies will be addressed through regular group meetings with all the reviewers and if needed additional consultation with the Writing Group and Health Professional Expert Advisory Group. The evidence review will be limited to a small selection of T\&CM therapies and where possible will only appraise existing systematic reviews (SR) and any clinical trials published after the date of the last SR. Other evidence will only be searched for and included if it is the best available evidence to answer the research question. No meta-analyses or modelling will be undertaken.

\section{Rapid scoping review}

The aim of the scoping review is to inform the PICO questions for the evidence review ${ }^{31}$ and to gather information about relevant modifying factors such as patient preferences, equity, economic and logistical considerations that will be needed when grading the recommendations for the Guidelines. ${ }^{38}$ Scoping reviews are useful in fields such as palliative care and T\&CM where there is emerging and heterogeneous evidence. ${ }^{46}$ It is anticipated that the available evidence will include a wide range of study designs and quality, along with various comparative interventions and outcomes. The nature, volume and direction of the evidence identified through the scoping review will inform the PICO questions and the search strategy for the evidence review.

Up to $20 \mathrm{~T} \& \mathrm{CM}$ interventions will be selected for stage 2, the evidence review. To assist the selection process, the scoping review will summarise information about the following:

i. T\&CM interventions commonly used by people with diabetes and/or people who have a life-threatening or life-limiting illness,

ii. T\&CM interventions commonly provided by caregivers, healthcare practitioners or palliative care services;

iii. Common problems and outcomes that people with diabetes at the end of life seek to address by using T\&CM;

iv. Other considerations such as safety, quality, cost, availability and feasibility of T\&CM interventions;

v. Relevance and applicability of the results to the Australian palliative care setting and the objectives of the Guidelines; and

vi. Quality of the research.

\section{Patient/problem/population}

The Guideline outlines common conditions and problems that people in Australia with diabetes experience. ${ }^{30}$ These are categorised according to the Palliative Care Outcomes Collaboration (PCOC) stages of stable, unstable, deteriorating and terminal, ${ }^{47}$ patients known to have pre-existing diabetes and those who develop diabetes; patients with Type 1 and Type 2 Diabetes; and common palliative care and diabetes problems (Table 2). There may however be reasons, other than the problems listed in the Guidelines, for why people with diabetes use T\&CM e.g. seeking more personal person-centred care, to support holistic healthcare needs, or to manage comorbidities. ${ }^{48,49}$ Therefore, the scoping review will also seek to identify other common or important reasons and problems for T\&CM use by this patient group that are not listed in Table 2.

\section{Intervention/comparison}

Given the vast number of potential T\&CM interventions, to assist the selection process of up to 20 interventions, the scoping review will gather information about interventions that: 
i. Are often used in Australia by this patient group, or commonly provided by their caregivers, healthcare practitioners or health services;

ii. Have higher quality scientific evidence about benefits or risks;

iii. Aim to address common problems that

iv. are experienced by people with diabetes at the end of life (especially when conventional care is ineffective, very costly, or low patient acceptability), or

v. aim to simultaneously address multiple problems or a symptom cluster experienced by people with diabetes and/or people at the end of life;

vi. Have safety concerns (e.g. pharmacokinetic interactions, hypoglycaemia);

vii. Have high costs (including opportunity costs); and

viii. Are logistically feasible to implement in the Australian palliative health care setting.

Table 2 Conditions and problems experienced by people with diabetes at end-of-life

Hyperglycaemia, hypoglycaemia, polyuria, oligouria, thirst

Microvascular disease (retinopathy and nephropathy)

Macrovascular disease (cardiovascular and cerebrovascular disease and intermittent claudication)

Neuropathy (peripheral and autonomic), pain

Active infection, sepsis, occult chronic infection

Depression, anxiety

Insomnia

Nausea, vomiting and anorexia

Acute dyspnoea

Cachexia

Delirium

\section{Outcomes}

The patient-relevant outcomes discussed in the Guidelines and its references include monitoring and stabilising blood glucose levels to minimise metabolic derangements and associated symptoms; maximising quality of life and comfort; and an emphasis on psychological, social and spiritual wellbeing. ${ }^{30}$ It is anticipated that the scoping review will identify other outcomes that are important to people with diabetes. Information about the relative importance of different outcomes may also be revealed. Potential outcomes will be classified as critical, important but not critical, or low importance. ${ }^{31}$

\section{Modifying factors}

Contextual information is essential for the decision-making process of patients, clinicians, health services and policy makers. ${ }^{36,38,39,50,51}$ The scoping review will also begin to collect information about important modifying factors such as costs, accessibility, feasibility of administration (e.g. in the hospice, hospital or home setting), quality control, clinical governance, patients' (and caregivers') preferences and values, and other social and economic considerations.

\section{Search strategy}

An iterative process will be used to search the literature. Searches will cease when data saturation is reached (i.e. no new themes or information are identified) and there is adequate information to answer the research questions and justify the selection of up to $20 \mathrm{~T} \& \mathrm{CM}$ interventions for the evidence review. Qualitative and quantitative studies are included and will be identified by first searching PubMed and selected EBSCOhost databases - Abstracts in Social Gerontology, Academic Search Complete, AHFS Consumer Medication Information, CINAHL Plus with Full Text, eBook Collection, Health Source - Consumer Edition, Health Source Nursing/Academic Edition, MEDLINE and PsycINFO. Combined, these on-line reference systems provide an extensive range of relevant index journals and grey literature. ${ }^{52}$ If data saturation is not achieved, other databases including the Allied and Complementary Medicine Database (AMED), ${ }^{53}$ Natural Medicines Comprehensive Database ${ }^{54}$ and Google Scholar will be searched. ${ }^{52,55}$ Bibliographies of published papers, and cluster searching of key authors will additionally inform the iterative process. Key words for the first search will focus on the utilization of T\&CM and will be based on three categories 1) diabetes and 2) palliative and end of life care and 3) common comorbidities, conditions or problems (Table 2). These are detailed in Additional File 1. Results from this initial search will inform subsequent searches until data saturation is reached.

\section{Data appraisal and synthesis}

Mixed methodology will be used to collate and synthesise the evidence from a range of study designs. Data will be organised on a spreadsheet. The CASP (Critical Appraisal Skills Programme) checklists will be used as a guide for rapidly rating the quality of the research. ${ }^{56}$

\section{Finalising the PICO questions}

The results of the rapid scoping review will be presented to the Writing Group and the Health Professional Expert Advisory Group in tabulated and narrative formats who will then jointly decide the final selection of the PICO questions to be answered by the T\&CM rapid evidence review. If more than 20 interventions are shortlisted, selection will be prioritised according to those that have 1) higher quality scientific evidence for safety and/or effectiveness; 2) use comparison interventions that are already recommended for use in this clinical setting; and 3) the studies evaluate outcomes that match the reasons why people with diabetes or T\&CM practitioners use the intervention or align with outcome important to people with diabetes.

\section{Rapid evidence review}

Having determined the PICO questions, a rapid evidence review will be conducted to inform the Guidelines revision. The aim is to identify and appraise the quality of evidence, risk of bias and confidence in the accuracy of estimated treatment effect sizes. ${ }^{57}$

\section{Search and selection methods}

In contrast to a comprehensive systematic review (SR) as outlined in the Cochrane Handbook for Systematic Reviews of Interventions, ${ }^{58}$ the rapid literature search will use a limited number of bibliographic databases and only include publications written in English. The 
search will begin by identifying SRs, followed by clinical trials published after the date of the SR. If the SR evidence is rated as low quality, then the search will be extended to include all clinical trials. Published research comparing T\&CM with active controls or placebo, followed by studies without controls will be sought. If significant uncertainty about benefits and risks remain, the inclusion criteria will be extended to include other study designs - observational studies, case controls, case series, case studies, evidence of traditional use (expert clinical opinion) and laboratory research. The Cochrane trial register, PubMed and EBSCOhost will initially be searched. Theses searches are likely to capture over $97 \%$ of published clinical trials for conventional medicine ${ }^{45}$ If needed, database searches of the Allied and Complementary Medicine Database (AMED).$^{53}$ Natural Medicines Comprehensive Database, ${ }^{54}$ AcuTrials $^{59}$ and Google Scholar will be searched. ${ }^{52,55}$ The bibliographies of published papers and cluster searching of key authors will also be used to identify additional evidence for inclusion in the review. A preliminary outline of the search terms can be found in the Additional File 1. At least seven key words for diabetes and co-morbidities, twenty key words for T\&CM interventions and ten key words for outcomes will be used. The final selection will be informed by the existing Guidelines, the agreed PICO questions and results from the scoping review. Keywords for study designs will aim to identify the highest quality evidence about each intervention. A PRISMA diagram will provide an overview of the search results.

\section{Quality assessment}

Studies will be critically appraised for the quality of the evidence and risk of bias by one reviewer using the following tools: AMSTAR checklist for systematic reviews, ${ }^{60,61}$ Cochrane Risk of Bias Tool for clinical trials,${ }^{58}$ and ROBINS-I for non-randomised intervention studies. ${ }^{62}$ The CONSORT extensions for reporting herbal medicinal interventions, ${ }^{63}$ non-pharmacologic treatment interventions, ${ }^{64}$ acupuncture interventions, ${ }^{65}$ non-inferiority and equivalence trials, ${ }^{66}$ and pragmatic trials ${ }^{67}$ will be used if relevant.

\section{Data synthesis}

The GRADE Evidence Profile and GRADEpro 3.6 software $^{33}$ will be used to collate, summarise and present the results to inform the Guidelines update. The results will be summarised in tabulated form using the GRADE Evidence to Decision (EtD) framework ${ }^{68}$ that incorporates the internationally recognised Cochrane Summary of Findings from systematic reviews on the effects of healthcare interventions. ${ }^{58}$ The EtD will be adapted to include the modifying factors-generalizability and applicability to the Australian healthcare setting - that are used in the Australian National Health and Medical Research Council's Body of Evidence Matrix. ${ }^{69}$

\section{Conclusions and recommendations}

The results of the evidence review - EtD framework and a narrative summary - will be presented to the Writing Group and the Health Professional Expert Advisory Group for consideration in the updated Guidelines.

\section{Discussion}

The proposed rapid review protocol addresses the time and resource constraints of the Guidelines developers; the need to develop robust methods for rapid reviews, ${ }^{3}$ and the ad-hoc consideration of T\&CM in many clinical guidelines. ${ }^{28,29,37} \mathrm{~A}$ two-step process has been proposed that begins with a scoping review to inform the PICO questions, followed by a literature search and appraisal of the evidence for up to 20 T\&CM interventions.

The primary purpose of this rapid review is to inform evidencebased recommendations for the Guidelines about the appropriate use of $\mathrm{T} \& \mathrm{CM}$ in for people with diabetes receiving palliative or end of life care. Given the project's constraints, an alternative option could be to undertake a systematic literature review that includes all relevant bibliographic databases, non-English publications and a meta-analysis for only one T\&CM intervention or one clinical problem. Although the results would be less prone to bias, they would also be much less useful for updating the Guidelines that require an evidence review about the safety and efficacy of a range of T\&CM interventions and problems that are relevant to this patient group.

Accepting there will be an increased risk of bias is a compromise for many rapid review protocols. ${ }^{7}$ The decision to include only one reviewer for the database searching, selecting eligible publications and appraising the quality of the evidence; restricting the evidence review to a limited number of T\&CM interventions, research published in English and a limited number of databases; and using the results of systematic reviews in preference to searching and appraising all clinical trials, will increase the risk of bias and the likelihood that relevant interventions and research may be missed or excluded. ${ }^{70}$

To help mitigate the limitations of the proposed study design, strategies such as using two reviewers to independently appraising a tenth of the papers, conducting broader database searches if needed, regular team meetings throughout, and publishing the protocol in advance of conducting the review have been employed. Furthermore, the impact of many of the methodological compromises of this protocol may not be substantial enough to warrant using more stringent methods. For example, an exhaustive search of all relevant databases may not substantially improve the reliability of the results. In a crosssectional analyses of 129 SRs, an exhaustive literature search of up to ten databases was not shown to significantly alter the estimated effect sizes compared to SRs of only two databases (EMBASE and Medline). ${ }^{71}$ Furthermore the inclusion of all possible trials of any quality may potentially increase the risk of bias due to the effects of low quality studies skewing the results. ${ }^{70}$

\section{Conclusion}

This rapid review protocol highlights its limitations and strengths, and the rationale for the proposed study design. The protocol addresses key areas of concern regarding the need for more systematic methods when formulating PICO questions for T\&CM interventions, and proposes methods for a rapid evidence synthesis to inform evidence based clinical guidelines. The incorporation of evidence based T\&CM into the Guidelines for the management of people with diabetes receiving palliative or end of life care has been proposed to improve patient-physician communication and contribute to coordinated continuity of care across multiple healthcare settings. It acknowledges the high use of T\&CM in Australia that includes adults with diabetes receiving palliative care or at the end of life, and the need for evidencebased guidance about the appropriate use of T\&CM.

The proposed rapid review will synthesise the highest quality evidence for T\&CM and provide meaningful information for the self-care agenda of people with diabetes who have a life-threatening or life-limiting illness, their caregivers, practitioners and healthcare 
services. ${ }^{72}$ In addition, it will identify important knowledge gaps to help guide research direction.

\section{Acknowledgements}

\section{Protocol review}

The protocol is endorsed by the Writing Group and the Health Professional Expert Advisory Group to the Revision of the Guidelines for Managing Diabetes at the End of Life, Australia. Centre for Nursing and Allied Health Research, Deakin University and Barwon Health. PO Box 281, Geelong, Victoria, 3220, Australia

\section{Authors' contributions}

$\mathrm{JH}, \mathrm{SA}$ and GD jointly developed the protocol. JH and SA wrote the manuscript, with editorial input from GD. All authors read and approved the final manuscript

\section{Acknowledgement}

The authors would like to thank their colleagues in the Writing Group and the Health Professional Expert Advisory Group to the Revision of the Guidelines for Managing Diabetes at the End of Life (listed below) for their input during the protocol development.

\section{Writing group}

Professor Trisha Dunning AM, Chair in Nursing and Director Centre for Quality and Patient Safety Research, Barwon Health Partnership, School of Nursing and Midwifery Deakin University.

Dr Susan Arentz, Adjunct Research Fellow, NICM, Western Sydney University.

Dr Gary Deed, Chair Diabetes Specific Interests Network, Royal Australian College of General Practice.

Dr Jennifer Hunter, Senior Research Fellow, NICM, Western Sydney University.

Dr Peter Martin, Professor of Clinical Communication and End of Life Care, School of Medicine, Deakin University and Palliative Medicine Physician, Barwon Health, Geelong.

Ms Sharyn Milnes, Curriculum and Research, i-Validate Project, Barwon Health

Dr Lisa Mitchell, Geriatrician, Barwon Health, Geelong.

Health Professional Expert Advisory Group:

Professor Trisha Dunning AM, Chair in Nursing and Director Centre for Quality and Patient Safety Research, Barwon Health Partnership, School of Nursing and Midwifery Deakin University.

Professor Craig Bennett, CEO Diabetes Victoria and Adjunct Professor Deakin University.

Caroline Ford, Group Manager Connected Living CommunityHome Support Services, Southern Cross Care (SA \& NT) Inc.,

Associate Professor Mark Kennedy, Department of General Practice, Melbourne Medical School, University of Melbourne; Medical Director, Northern Bay Health.

Professor Geoffrey Mitchell, General Practice and Palliative Care, Primary Care Clinical Unit, Faculty of Medicine, The University of Queensland.
Associate Professor Neil Orford, Medical Director Intensive Care Unit, University Hospital Barwon Health, Geelong.

\section{Conflicts of interest}

The authors declare that they have no competing interests. All three authors are members of the Writing Group to the Revision of the Guidelines for Managing Diabetes at the End of Life, Australia.

\section{Funding}

The project will be partially funded by NICM, Western Sydney University, and pro-bono contributions from all three the authors. As a medical research institute, NICM receives research grants and donations from foundations, universities, government agencies, individuals and industry. Sponsors and donors provide untied funding for work to advance the vision and mission of NICM. The project that is the subject of this article was not undertaken as part of a contractual relationship with any donor or sponsor.

\section{References}

1. McIntosh HM, Calvert J, Macpherson KJ, The Healthcare improvement scotland evidence note rapid review process: providing timely, reliable evidence to inform imperative decisions on healthcare. Int J Evid Based Health. 2016;14(2):95-101.

2. Polisena J, Garritty C, Kamel C, et al. Rapid review programs to support health care and policy decision making: a descriptive analysis of processes and methods. Syst Rev. 2015;4:26.

3. Tricco AC, Antony J, Zarin W, et al. A scoping review of rapid review methods. BMC Med. 2015;13:224.

4. Featherstone RM, Dryden DM, Foisy M, et al. Advancing knowledge of rapid reviews: an analysis of results, conclusions and recommendations from published review articles examining rapid reviews. Syst Reviews. 2015;4:50.

5. Grant MJ, Booth A. A typology of reviews: an analysis of 14 review types and associated methodologies. Health info Libr J. 2009;26(2):91-108.

6. Petticrew M, Roberts H. Systematic reviews in the social sciences: A practical guide. USA: John Wiley, Sons; 2008. p. 1-336.

7. Ganann R, Ciliska D, Thomas H. Expediting systematic reviews: methods and implications of rapid reviews. Implement Sci. 2010;5:56.

8. https://www.aihw.gov.au/

9. Dunning T, Duggan N, Savage S. Caring for people with diabetes at the end of life. Curr Diab Rep. 2016;16:103.

10. Klingler C, in der Schmitten J, Marckmann G. Does facilitated advance care planning reduce the costs of care near the end of life? Systematic review and ethical considerations. Palliat Med. 2016;30(5):423-433.

11. Wieland LS, Manheimer E, Berman BM. Development and classification of an operational definition of complementary and alternative medicine for the Cochrane collaboration. Altern Ther Health Med. 2011;17(2):5059 .

12. http://www.nicm.edu.au/health_information/information_for consumers/understanding_cm

13. NCCAM. What is CAM? USA: United States National Institute of Health. USA: Springer; 2009.

14. Chang Hy, Wallis M, Tiralongo E. Use of complementary and alternative medicine among people living with diabetes: literature review. $J A d v$ Nurs. 2007;58(4):307-319. 
15. Manya K, Champion B, Dunning T. The use of complementary and alternative medicine among people living with diabetes in Sydney. BMC complement Altern Med. 2012;12:1.

16. Nahin RL, Byrd-Clark D, Stussman BJ, et al. Disease severity is associated with the use of complementary medicine to treat or manage type-2 diabetes: data from the 2002 and 2007 National health interview survey. BMC complement Altern Med. 2012;12:193.

17. Dain AS, Bradley EH, Hurzeler R, et al. Massage, music, and art therapy in hospice: results of a national survey. J Pain Symptom Manage. 2015;49(6):1035-1041.

18. Johnson EL, O'Brien D. Integrative therapies in hospice and home health: introduction and adoption. Home Healthc Nurse. 2009;27(2):75-82.

19. Leng G. Use of acupuncture in hospices and palliative care services in the UK. Acupunct Med. 2013;31(1):16-22.

20. Necyk C, Zubach-Cassano L. Natural health products and diabetes: a practical review. Can J Diabetes. 2017;41(6):642-647.

21. Bishop FL, Yardley L, Lewith GT. A systematic review of beliefs involved in the use of complementary and alternative medicine. $J$ Health Psychol. 2007;12:851-867.

22. Lin V, Canaway R, Carter B, et al. Room for improvement: complementary therapy users and the Australian health system. Health expectations: an international journal of public participation in health care and health policy. 2013;18(5):1451-1462.

23. Lin V, Canaway R, Carter B. Interface, interaction and integration: how people with chronic disease in Australia manage CAM and conventional medical services. Health expect. 2014;18(6):2651-2665.

24. Dunning T, Duggan N, Savage S, et al. Diabetes and end of life: ethical and methodological issues in gathering evidence to guide care. Scand $J$ Caring Sci. 2013;27(1):203-211.

25. Poulson J. The management of diabetes in patients with advanced cancer. J pain symptom manage. 1997;13(6):339-346.

26. Quinn K, Hudson P, Dunning T. Diabetes management in patients receiving palliative care. J pain symptom manage. 2006;32(3):275-286.

27. Vandenhaute V. Palliative care and type II diabetes: a need for new guidelines? Am J Hos Palliat Med. 2010;27(7):444-445.

28. Ernst E. Assessments of complementary and alternative medicine: the clinical guidelines from NICE. Int J Clin Pract. 2010;64(10):1350-1358.

29. Lorenc A, Leach J, Robinson N. Clinical guidelines in the UK: Do they mention Complementary and alternative medicine (CAM) - Are CAM professional bodies aware? European Journal of Integrative Medicine. 2014;6(2):164-175.

30. Dunning T, Martin P, Savage S, et al. Guidelines for managing people with diabetes at the end of life: Final report. 2010. p. 1-235.

31. Guyatt GH, Oxman AD, Kunz R, et al. GRADE guidelines: 2. Framing the question and deciding on important outcomes. $J$ clin Epidemiol. 2011;64(4):395-400.

32. Muche-Borowski C, Selbmann H, Nothacker M, et al. AWMF guidance manual and rules for guideline development. german association of the scientific medical societies; 2012.

33. GRADEpro GDT: GRADEpro Guideline Development Tool. Inc, McMaster University, Canada: Evidence Prime; 2015.

34. Hunter J, Leach M, Braun L, An interpretive review of consensus statements on clinical guideline development and their application in the field of traditional and complementary medicine. BMC Complement Altern Med. 2017;17(1):116.
35. https://www.nice.org.uk/process/pmg6/chapter/introduction

36. http://www.who.int/iris/handle/10665/68925

37. Robinson N, Lui J, Lee MS. Clinical guidelines: The way for best practice. European Journal of Integrative Medicine. 2014;6(2):133-134.

38. Andrews JC, Schunemann HJ, Oxman AD, et al. GRADE guidelines: 15. Going from evidence to recommendation-determinants of a recommendation's direction and strength. $J$ clin epidemiol. 2013;66(7):726-735.

39. Andrews J, Guyatt G, Oxman AD, et al. GRADE guidelines: 14. Going from evidence to recommendations: the significance and presentation of recommendations. J clin epidemiol. 2013;66(7):719-725.

40. Oxman AD, Lavis JN, Fretheim A, et al. SUPPORT Tools for evidenceinformed health Policymaking (STP) 17: Dealing with insufficient research evidence. Health Res Policy Syst. 2009;7(1):S17.

41. Petitti DB, Teutsch SM, Barton MB, et al. Update on the methods of the U.S. preventive services task force: insufficient evidence. Annals of internal medicine. 2009;150(3):199-205.

42. Woolf S, Schunemann HJ, Eccles MP, et al. Developing clinical practice guidelines: types of evidence and outcomes; values and economics, synthesis, grading, and presentation and deriving recommendations. Implement Sci. 2012;7:61.

43. Edejer TT. Improving the use of research evidence in guideline development: 11. Incorporating considerations of cost-effectiveness, affordability and resource implications. Health Res Policy Syst. $2006 ; 4: 23$

44. Thornton J, Alderson P, Tan T, et al. Introducing GRADE across the NICE clinical guideline program. J Clin Epidemiol. 2013;66(2):124-131.

45. Royle P, Milne R. Literature searching for randomized controlled trials used in Cochrane reviews: rapid versus exhaustive searches. Int J Technol Assess Health Care. 2003;19(4):591-603.

46. Colquhoun HL, Levac D, Brien KK, et al. Scoping reviews: time for clarity in definition, methods, and reporting. J Clinical Epidemiol. 2014;67(12):1291-1294.

47. Eagar K, Watters P, Currow DC, et al. The Australian Palliative Care Outcomes Collaboration (PCOC)-measuring the quality and outcomes of palliative care on a routine basis. Aust Health Rev. 2010;34(2):186-192.

48. Barth J, Schafroth L, Witt CM. Overlap and differences between patient and provider expectations for treatment outcomes: the case of acupuncture. J Pain. 2016;17(6):685-693.

49. Canaway R, Manderson L, Oldenburg B. Perceptions of benefit of complementary therapy use among people with diabetes and cardiovascular disease. Forsch Komplementmed. 2014;21(1):25-33.

50. Truman BI, Smith Akin CK, Hinman AR, et al. Developing the Guide to Community Preventive Services-overview and rationale. The Task Force on Community Preventive Services. Am J Prev Med. 2000;18:18-26.

51. NICE. Developing NICE guidelines: the manual. Process and methods guide. National institute for health and care excellence, London, UK: Springer; 2014.

52. Cogo E, Sampson M, Ajiferuke I, et al. Searching for controlled trials of complementary and alternative medicine: a comparison of 15 databases. Evid Based Complement Alternat Med. 2011;2011:858246.

53. Vardell E. AMED: The Allied and Complementary Medicine Database. Med Ref Serv Q. 2016;35(4):434-439.

54. Natural Medicines Comprehensive Database Stockton. Therapeutic Research Faculty, California, USA: Springer; 2017. 
55. Shekelle PG, Morton SC, Suttorp MJ, et al. Challenges in systematic reviews of complementary and alternative medicine topics. Ann intern med. 2005;142(12):1042-1047.

56. http://www.casp-uk.net/casp-tools-checklists

57. Balshem H, Helfand M, Schünemann HJ, et al. GRADE guidelines: 3. Rating the quality of evidence. J clin epidemiol. 2011;64(4):401-406.

58. www.handbook.cochrane.org

59. Marx BL, Milley R, Cantor DG, et al. AcuTrials ${ }^{\circledR}$ : an online database of randomized controlled trials and systematic reviews of acupuncture. BMC Complement Altern Med. 2013;13:181.

60. Pollock M, Fernandes RM, Hartling L. Evaluation of AMSTAR to assess the methodological quality of systematic reviews in overviews of reviews of healthcare interventions. BMC med res methodol. 2017;17(1):48.

61. Shea BJ, Hamel C, Wells GA, et al. AMSTAR is a reliable and valid measurement tool to assess the methodological quality of systematic reviews. J clin epidemiol. 2009;62(10):1013-1020.

62. Sterne JA, Hernan MA, Reeves BC, et al. ROBINS-I: a tool for assessing risk of bias in non-randomised studies of interventions. $B M J$. 2016;355:i4919.

63. Gagnier JJ, Boon H, Rochon P, et al. Reporting randomized, controlled trials of herbal interventions: an elaborated CONSORT statement. Ann intern med. 2006;144(5):364-367.

64. Boutron I, Moher D, Altman DG, et al. Extending the CONSORT statement to randomized trials of nonpharmacologic treatment: explanation and elaboration. Ann intern med. 2008;148(4):295-309.
65. MacPherson H, Altman DG, Hammerschlag R, et al. Revised STandards for Reporting Interventions in Clinical Trials of Acupuncture (STRICTA): extending the CONSORT statement. PLoS med. 2010;7(6):e1000261.

66. Piaggio G, Elbourne DR, Pocock SJ, et al. Reporting of noninferiority and equivalence randomized trials: extension of the CONSORT 2010 statement. JAMA. 2012;308(24):2594-604.

67. Zwarenstein M, Treweek S, Gagnier JJ, et al. Improving the reporting of pragmatic trials: an extension of the CONSORT statement. BMJ. 2008;337:a2390.

68. Neumann I, Brignardello-Petersen R, Wiercioch W, et al. The GRADE evidence-to-decision framework: a report of its testing and application in 15 international guideline panels. Implement sci. 2016;11:93.

69. Hillier S, Grimmer-Somers K, Merlin T, et al. FORM: an Australian method for formulating and grading recommendations in evidence-based clinical guidelines. BMC med res methodol. 2011;11:23.

70. Egger M, Juni P, Bartlett C, et al. How important are comprehensive literature searches and the assessment of trial quality in systematic reviews? Health Technol Assess. 2003;7(1):1-76.

71. Hartling L, Featherstone R, Nuspl M, et al. The contribution of databases to the results of systematic reviews: a cross-sectional study. BMC med res methodol. 2012 6;6(1):127.

72. Tallon D, Chard J, Dieppe P. Relation between agendas of the research community and the research consumer. Lancet. 2000;355(9220):20372040 . 